Provided for non-commercial research and education use. Not for reproduction, distribution or commercial use.

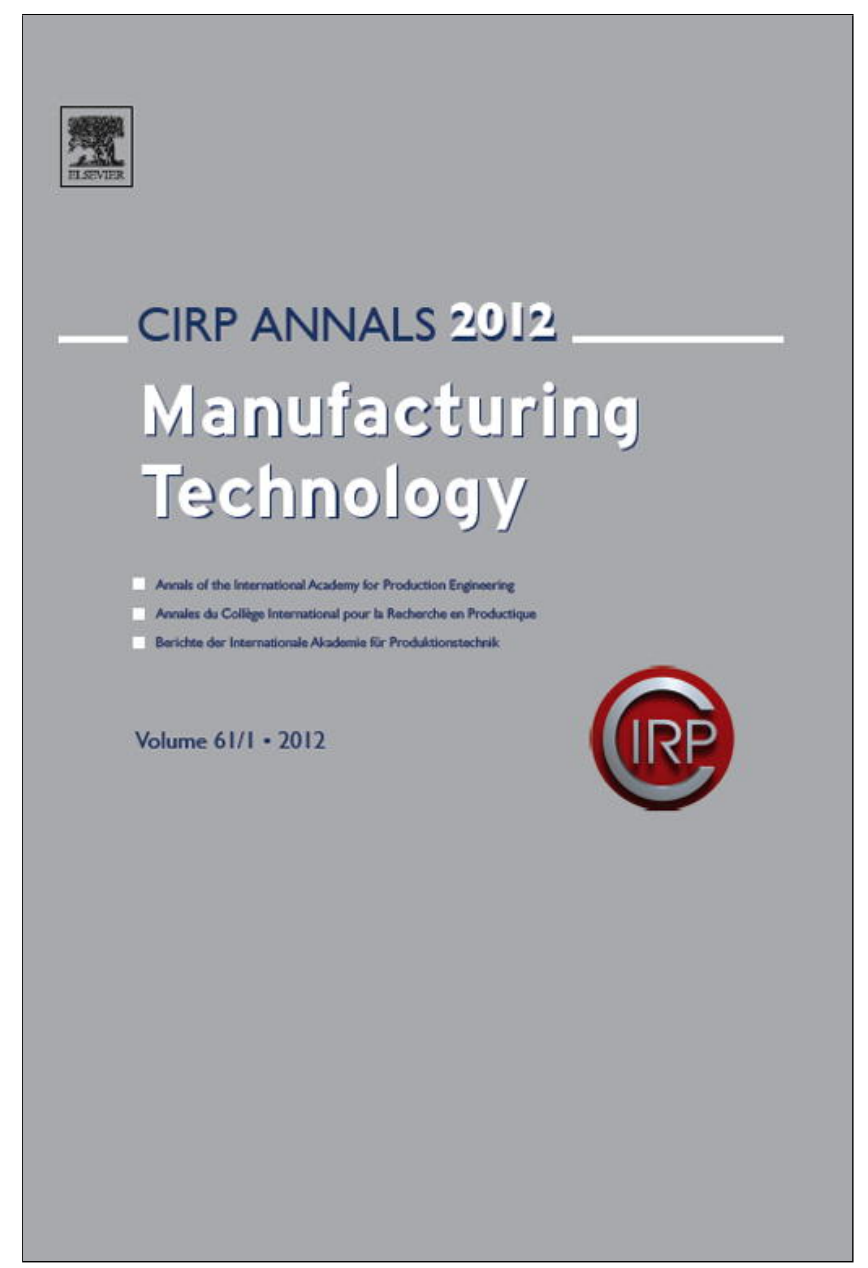

This article appeared in a journal published by Elsevier. The attached copy is furnished to the author for internal non-commercial research and education use, including for instruction at the authors institution and sharing with colleagues.

Other uses, including reproduction and distribution, or selling or licensing copies, or posting to personal, institutional or third party websites are prohibited.

In most cases authors are permitted to post their version of the article (e.g. in Word or Tex form) to their personal website or institutional repository. Authors requiring further information regarding Elsevier's archiving and manuscript policies are encouraged to visit:

http://www.elsevier.com/copyright 


\title{
Five-axis milling tool path generation with dynamic step-over calculation based on integrated material removal simulation
}

\author{
Bert Lauwers (1)*, Denys Plakhotnik \\ Department of Mechanical Engineering, Division PMA, K.U. Leuven, Leuven, Belgium
}

A R T I C L E I N F O

\section{Keywords:}

Computer Aided Manufacturing (CAM)

Milling

Toolpath

\begin{abstract}
A B S T R A C T
This paper describes the development of a five-axis tool path generation algorithm for the machining of complex shaped parts using flat-end tools. In order to reduce the machining time and to ensure optimal quality, the step-over is optimized in each tool contact point. This step-over calculation is obtained through the integration of a newly developed algorithm for material removal simulation. The latter is performed by calculating swept volumes which represent tool motions interpolated according to the machine kinematics. The developed algorithm has been implemented for contour like (spiral) strategies and validated through the machining of an industrial example.
\end{abstract}

(C) 2012 CIRP.

\section{Introduction}

Five-axis milling is a widely used technique to machine complex shaped parts such as impellers, mould cavities or any other mechanical part. Efficient and productive machining is obtained when flat-end or toroidal tools are applied [1,2].

In general, tool path generation algorithms implement openloop procedures that assign tool path patterns and tool orientations independently from each other. Tool path tracks are created on a surface by either projection methods (e.g. drive surface or cutting plane methods) or parametric space methods in which a tool path pattern is fixed and does not depend on the tool inclination strategy which is applied afterwards. Once the tool path track (=sequence of tool positions) is known, the tool orientation can be calculated to optimize the material removal (increasing machining strip width) or to avoid gouging and collision [1-4].

The disconnection between tool path pattern generation and tool posture optimization (especially tool inclination) has inevitably attracted attention of researchers because it results in machined surfaces with varying and non-controllable scallop height. In [4], it was already proposed to use the estimated machining strip width (calculated as the intersection of an elliptic profile, being the projection of the tool bottom into a plane perpendicular to the feed direction) to generate non-isoparametric tool paths with constant scallop height for flat-end milling tools. Other researchers [5-7] have further developed this method by implementing more accurate methods for the calculation of the machining strip width. However, all these methods calculate the machining strip width at a certain location based on a single tool posture, leading to substantial errors in scallop prediction. As explained in Fig. 1, the calculation of the resulting scallop in a given plane (perpendicular to the feed direction) is a result of multiple tool positions along the tool path track. The tool motion before and

\footnotetext{
* Corresponding author.
}

after a particular posture influences the shape of removed material, as also explained in $[8,9]$.

Research on incorporating more accurate material removal simulation algorithms within tool path generation is needed. Today, they have been implemented for ball-end tools [10,11], while the integration of removal simulation methods for flat-end tools are not embedded within tool path generation because they are still computationally demanding and cannot be efficiently performed in an iterative way.

This paper describes the development (implementations in Java, including wrapping visualization classes for VTK (vtk.org)) of a five-axis tool path generation algorithm, where the step-over between tracks is optimized based on an integrated material removal simulation for end-mills. The algorithm (described in Section 3 ) is based on a more accurate and realistic machining strip width calculation. Therefore, a new material removal simulation algorithm, taking the machine kinematics into account, has been developed (Section 2). The part surfaces are represented as a faceted model (STL-file). The industrial validation of the algorithm is explained in Section 4.

\section{Material removal simulation}

As explained above, an accurate calculation of the machining strip width is essential for a more optimized tool path generation. The developed algorithm is based on an already generated tool path (or a series of tool path segments), the part surface, the unmachined surface, the tool geometry (flat-end tool radius), and the machine kinematics.

As an example, Fig. 2a shows a number of tool postures arranged along a programmed tool path track (Section 3 explains how this is generated). Evenly spaced points are distributed on the tool path (Fig. 2b). In each of these points, a local coordinate system (LCS, $x$-axis along the feed vector, $z$-axis along the surface normal) and a plane (called swept section plane) defined by the $y z$-axes of the LCS, are constructed. 

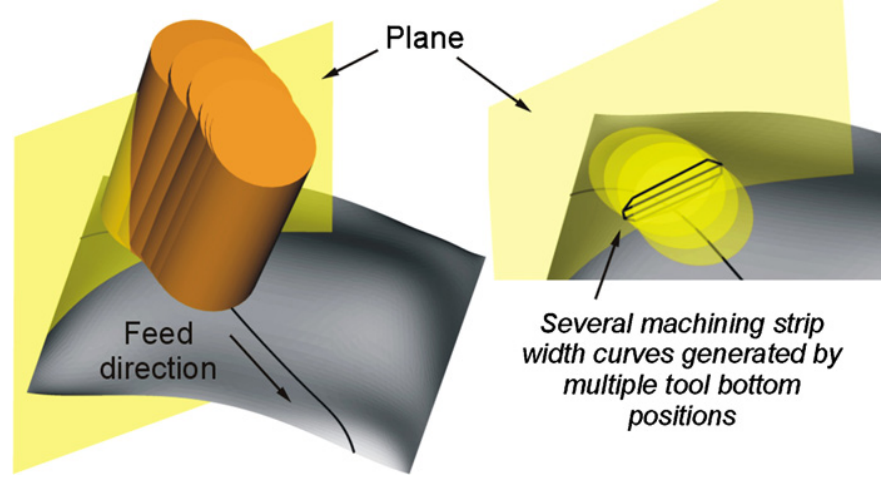

Several machining strip width curves generated by multiple tool bottom positions

Fig. 1. Effect of a sequence of tool postures on the machined area.

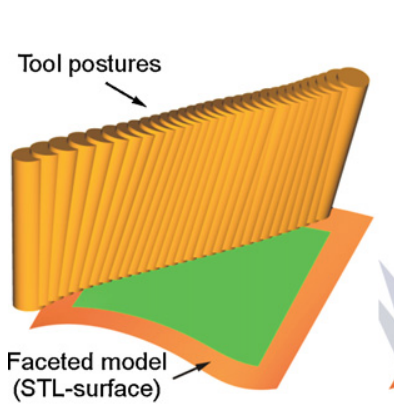

(a)

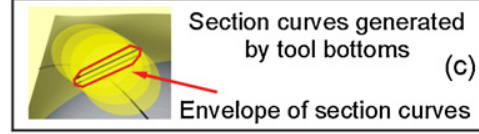

Swept section planes

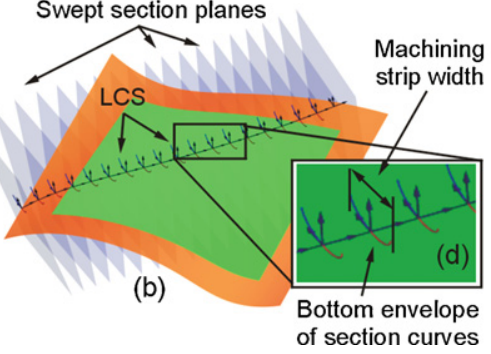

Fig. 2. Calculation of the machining strip width.

In each of these planes, the machining strip width is calculated. To do this, the complete tool movement that can influence the shape of a machined profile within a section plane is taken into account (see also Fig. 1). In order to increase precision, intermediate tool postures are generated laying between the original tool postures generated from the tool path generation algorithm (see Section 3). An interpolation of tool postures ( $x, y, z, i$, $j, k$ ) will result in machining inaccuracies (due to the kinematics of the machine). Therefore, a linear interpolation of the machine coordinates is performed. The latter is different and innovative to many other existing algorithms for material removal simulation. Inverse kinematic transformation was implemented only for the rotary axes because the machine used within this research supports tool center management functionality which performs translation offsets inside the $\mathrm{CNC}$ unit. Therefore, a tool end point $(x, y, z)$ moves linearly from the start position to the end position, as given in Eq. (1). The tool orientation vectors $(i, j, k)$ of the interpolated tool postures are derived from a forward kinematics calculation (Eq. (2)) of the rotary axes values of the milling machine. It is assumed that the rotary axes of the machine change linearly between the start and end positions, as given in Eq. (3). Since tool orientations $(i, j, k)$ are defined in every initial tool posture, inverse kinematic transformations given in Eqs. (4) and (5) are used to find the rotary axes values $(B(0), B(1), C(0), C(1))$ in the start and end positions.

$\mathbf{C L}(t)=\mathbf{C L}(0)+t(\mathbf{C L}(1)-\mathbf{C L}(0))$

$[i j k]^{T}=\operatorname{RotB}(B(t)) \times \operatorname{RotC}(C(t)) \times\left[\begin{array}{lll}0 & 0 & 1\end{array}\right]^{T}$

where RotB and RotC represent the coordinate transformation matrices associated with the $B$ and $C$ rotary axes.

$B(t)=B(0)+t(B(1)-B(0))$

$C(t)=C(0)+t(C(1)-C(0))$

$B=-\arctan 2\left[-2 \sqrt{k-k^{2}}, 2 k-1\right]$
$C=-\arctan 2\left[-i(k-1)+j \sqrt{2 k-2 k^{2}}, i \sqrt{2 k-2 k^{2}}+j(k-1)\right]$

The intersections between a swept section plane and the different tool bottoms (as the tool moves) result in a number of swept section curves (Fig. 2c). By combining the different swept section curves, an overall polygon is derived representing the shape of material removed by the tool bottom within the section plane. The machining strip width (Fig. 2d) can now be calculated by limiting the bottom bound of the polygon by the un-machined surface (defined by the stock).

Machining strip widths are calculated in each section plane and are used in Section 3 for optimized tool path generation. In addition the material removed by a complete tool move is calculated as well. To perform this, boxes are created by constructing two planes in addition to the swept section planes (Fig. 3 ). The extra planes pass through the end points of machining strip. A part of a surface that is inside a swept box is marked as machined. Technically, all vertices of the faceted model (STL mesh) that are inside the boxes are marked as machined. Fig. 3 (right) shows the machined region for one tool path track.

\section{Generation of tool path tracks with dynamic step-over}

The generation of tool path patterns is done according to a contour-like strategy, as it results in a more efficient machining with respect to smoothness and less non-cutting movements. A series of tool path loops are generated in an iterative manner by reconsidering un-machined areas after each loop. As an example, results can be seen on Fig. 9. However, the developed approach can be implemented for any kind of strategy such as zig or zig-zag.

The developed algorithm starts with the generation of a first initial outer loop (machining is done from outside to inside). Based on a user defined entry point and a direction, the end-point of the first tool path segment (line) is calculated (Fig. 4). The direction of the first segment and length of the segments can be chosen, but it should be defined based on part properties (size, curvature) and the tool radius. Within this research, the initial length of a tool path segment was set to $50 \%$ of the tool radius and the direction (angle between de the first tool path segment and the border tangent) was set to $60^{\circ}$.

Following tool path segments are added to the previous segment(s) as such that the distance to the border of the unmachined area remains more or less constant (e.g. distance $\sim 50 \%$
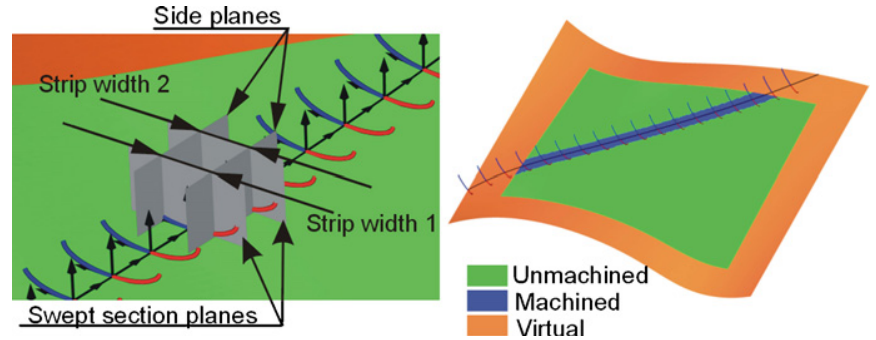

Fig. 3. Calculation of the removed material.

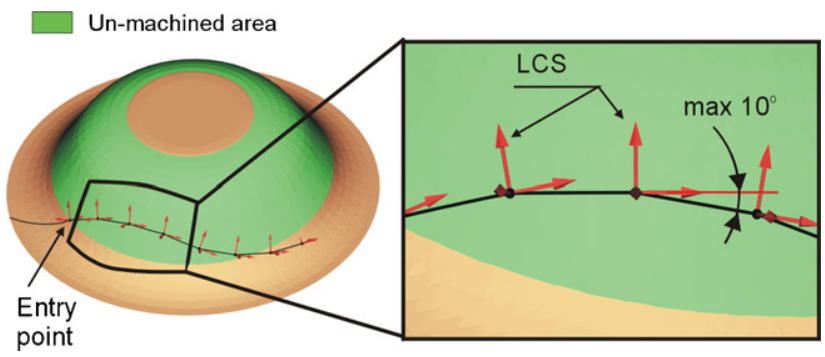

Fig. 4. Generation of initial (smooth) tool path loop. 
of the tool radius). After adding a segment (initially following the same direction of the previous segment), the distance from the end point of that segment to the border of the un-machined area in the direction perpendicular to this tool path segment is calculated. If this distance sufficiently differs from the pre-set distance, the segment is rotated around the surface normal at the start point of the segment in order to meet the target distance. In order to guarantee a smooth tool path, there is a limit imposed on a feasible angle deviation between successive tool path segments (e.g. $10^{\circ}$, Fig. 4). This limit sometimes causes that the algorithm encounters a non-resolvable scenario in which the end point further deviates from the border than allowed. In such a case, the algorithm backtracks to the previous segments by recursively changing angles between segments. Loop generation ends when a next segment is located in the vicinity of the beginning of the loop. Once the initial loop is generated, tool orientations are assigned to each tool contact point. Since tool orientations are assigned independently from the loop generation, various tool inclination strategies can be implemented. Within this research, a dynamic tool inclination strategy, earlier developed in [2], has been reimplemented. Once the tool postures are known, the machining strip width (blue and red curve in Fig. 5), can be calculated (see Section 3).

By analyzing the machining strip width (Fig. 5 (right)), it can be observed that in some regions, the tool path goes too much into the un-machined zone, leaving rest material at the border, while in other zones, the machining efficiency is very low (blue curve almost at border line of the un-machined area). This means that parts of the tool path should be adjusted either to better utilize the machining strip width (zone with low efficiency on Fig. 5) or become closer to the border in order to fully machine the unmachined area at the border side.

Tool path adjustments are done by an iterative sliding of the end points of tool path segments across the triangles of the part surface and in a direction perpendicular to the feed direction (Fig. 6). Based on the position of the initial tool path loop, possible shifts for tool path segments are first calculated and are represented in Fig. 6 as an inner and an outer range (series of dots). A point of the inner range is calculated by moving a tool path point over a distance equals the distance of the lower red curve of the machine strip width (Fig. 5) to the border of the un-machined area or a maximum
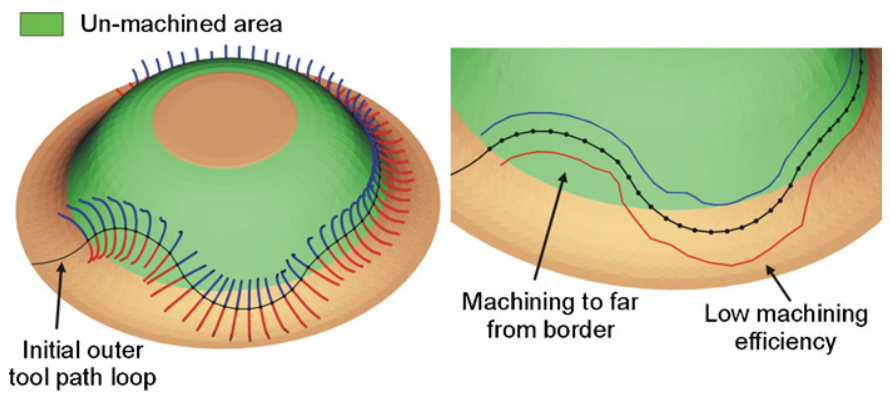

Fig. 5. Tool path adjustment based on estimated machining strip.

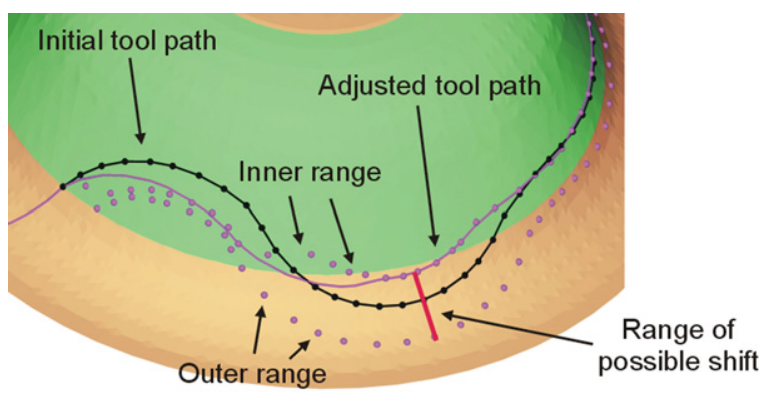

Fig. 6. Adjustment of the tool path within an inner and outer range.
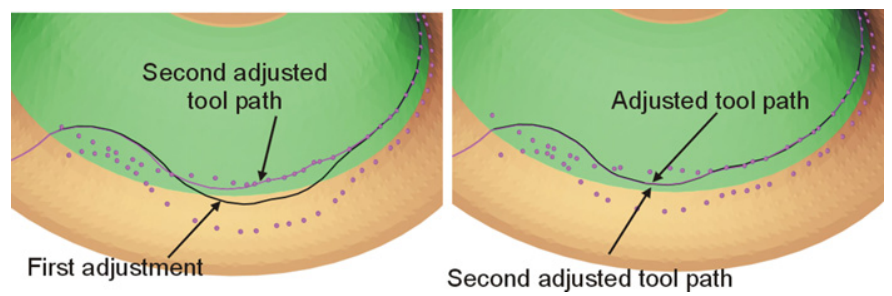

Fig. 7. Next iterations in tool path adjustments.
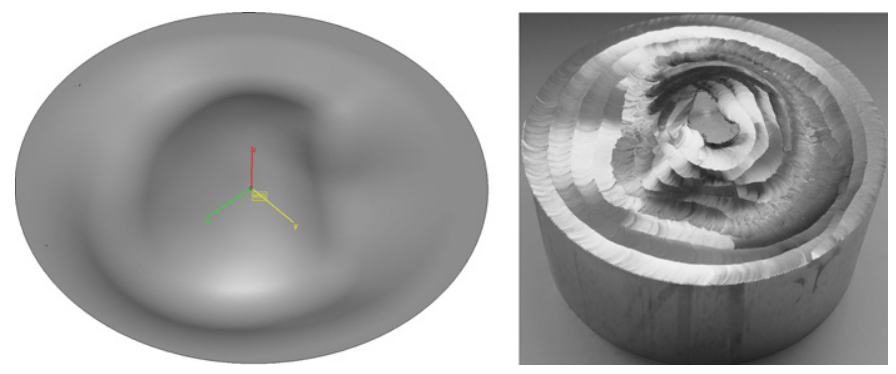

Fig. 8. Industrial example: (a) faceted model, (b) machined part.

percentage of the tool radius (in this case $50 \%$, set to avoid extreme shifts). A point of the outer range is calculated by moving a tool path point over a distance which is a percentage (in this case $66 \%$ ) of the tool radius. As an example, the red line in Fig. 6 shows the possible shift for that particular tool path point. Tool path adjustments are made possible by increasing the maximum angle between tool path segments or by changing the start direction at the entry point.

Fig. 7 shows the second and third iteration for tool path adjustment. After every adjustment, new swept sections and possible shifts for tool path changes are computed. The algorithm attempts to move the tool path to the inner range because this results in the most efficient utilization of machining strip width. Loop adjustment stops when there is no or little (below certain threshold) difference between two successive adjustments. The last adjustment is taken as the final tool path loop.

After the outer tool path loop has been generated, successive tool path loops (moving inwards) are generated following the same procedure. Based on the accurate material removal simulation, the border of the un-machined regions is updated. The last tool path segment (of the previous tool path loop) is taken as a start segment for the next tool path loop. Tool path generation terminates if machining efficiency drops below a certain level or the curvature of a loop reaches a critical value. The machining efficiency is defined as the amount of machined area per unit tool path length (see also Section 4).

\section{Validation: industrial example}

To prove the efficiency of the developed algorithm, the faceted model (size $\sim 100 \mathrm{~mm}$ in diameter) shown in Fig. 8, a die to produce skull implants by hydroforming, has been machined on a five-axis milling machine. The triangulation tolerance was set to $0.01 \mathrm{~mm}$ with maximal edge length of $0.5 \mathrm{~mm}$, resulting in 103k triangles. A flat-end milling tool ( $10 \mathrm{~mm}$, HSS) was used.

The length of the tool path segments has been limited to $2.5 \mathrm{~mm}$ (50\% tool radius). A maximal allowed scallop height was set to $0.17 \mathrm{~mm}$. The whole tool path consists of 9 loops. Fig. 9 depicts simulation of changes in the machined area after each loop.

Quantitative results from the tool path generation are summarized in Table 1 . The loop lengths gradually decrease approaching the core of the part. The efficiency (as defined above) varies at different steps, but drastically drops at the last step because less stock can be reached by a smooth-constrained tool 

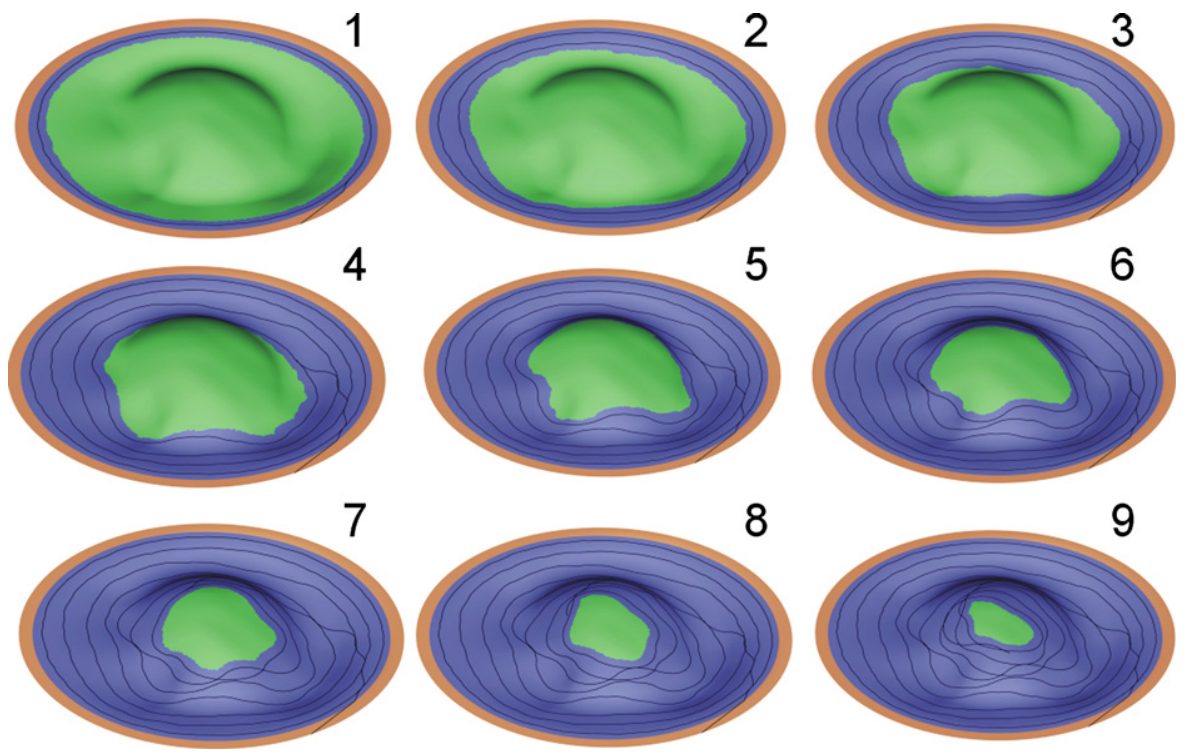

Fig. 9. Tool path generation steps for the part presented in Fig. 8.

Table 1

Results of the tool path generation calculations.

\begin{tabular}{|c|c|c|c|c|c|c|c|c|c|}
\hline $\mathrm{A}$ & 1 & 2 & 3 & 4 & 5 & 6 & 7 & 8 & 9 \\
\hline B & 301 & 281 & 250 & 218 & 186 & 163 & 140 & 111 & 115 \\
\hline C & 1275 & 1318 & 1344 & 1185 & 809 & 583 & 436 & 351 & 231 \\
\hline D & 4.24 & 4.70 & 5.38 & 5.43 & 4.34 & 3.58 & 3.11 & 3.16 & 2.01 \\
\hline
\end{tabular}

A: loop number.

B: loop length $[\mathrm{mm}]$

C: machined area per loop $\left[\mathrm{mm}^{2}\right]$.

D: area removal ratio per loop $\left[\mathrm{mm}^{2} / \mathrm{mm}\right]$

path when an insignificantly small area of un-machined area is left. Therefore, the area removal ratio can be used as a trigger to halt tool path generation in fully automated systems. For the example of Fig. 9, $279 \mathrm{~mm}^{2}$ remained un-machined at the top and should be machined by another strategy.

The part has been machined in aluminum (Fig. 8b). The shape of the un-machined island is similar to the shape from the machining simulation. Scallop heights have been measured along 7 directions (perpendicular to the border) distributed over the part. The measured heights are between 150 and $180 \mu \mathrm{m}$, which are in the range of the pre-set value of $170 \mu \mathrm{m}$.

The developed strategy was also compared to a five-axis tool path generation strategy available within a commercial CAM system, based on faceted models. A zig-zag strategy had to be chosen, as it was the only strategy allowing the calculation of a step-over based on a given scallop value. For the same tool path generation settings (scallop, tool, inclination angle $\left(5^{\circ}\right.$ and adaptable to avoid gouging) were chosen. The length of the resulting tool path is $17261 \mathrm{~mm}$, which is certainly much higher than $1765 \mathrm{~mm}$ (sum of the 9 tool path loops, Table 1 ).

\section{Conclusions}

This paper described the development and implementation of a new tool path planning and generation strategy for five-axis machining. Compared to commercial available strategies, the tool path length can substantially be shortened by optimizing the stepover between adjacent tool path loops. A novel method to calculate the material removal, including machine kinematics information, is used not only for computing the step-over but also for predicting areas of machined areas. The scope of application that can benefit from the developed method ranges from finishing and semifinishing to even rough milling based on morphing technology as described in [12].

\section{Acknowledgments}

This research has been funded through the EU-project NEXT (NMP-IP-011815) and FWO project G.0505.07N (Research Foundation - Flanders).

\section{References}

[1] Kruth J-P, Klewais P (1994) Optimization and Dynamic Adaptation of the Cutter Inclination During Five-axis Milling of Sculptured Surfaces. Annals of the CIRP 43(1):443-448.

[2] Lauwers B, Kiswanto G, Kruth J-P (2003) Development of a Five-axis Milling Tool Path Generation Algorithm Based on Faceted Models. Annals of the CIRP 52(1):85-88.

[3] Vickers GW, Quan KW (1989) Ball Mills Versus End Mills for Curved Surface Machining. ASME Journal of Engineering in Industry 111:22-26.

[4] Lee Y-S (1999) Adaptive Tool Path Planning for 5-axis Sculptured Surface Machining by Machining Strip Evaluation, SSM '98:. Proceedings of the IFIP TC5 WG5.3 International Conference on Sculptured Surface Machining, 351-360.

[5] Chiou C-J, Lee Y-S (2002) A Machining Potential Field Approach to Tool Path Generation for Multi-axis Sculptured Surface Machining. Computer-Aided Design 34:357-371.

[6] Tournier C, Duc E (2005) Iso-scallop Tool Path Generation in 5-axis Milling. The International Journal of Advanced Manufacturing Technology 25:867-875.

[7] Barakchi Fard M, Feng H-Y (2009) Effect of Tool Tilt Angle on Machining Strip Width in Five-axis Flat-end Milling of Free-form Surfaces. The International Journal of Advanced Manufacturing Technology 44:211-222.

[8] Chiou C-J, Lee Y-S (1999) A Shape-generating Approach for Multi-axis Machining G-buffer Models. Computer-Aided Design 31:761-776.

[9] Sarma R (2000) Flat-ended Tool Swept Sections for Five-axis NC Machining of Sculptured Surfaces. Journal of Manufacturing Science and Engineering 122(1):158-165.

[10] Lazoglu I, Manav C, Murtezaoglu Y (2009) Tool Path Optimization for Free Form Surface Machining. Annals of the CIRP 58(1):101-104.

[11] López de Lacalle L, Lamikiz A, Sánchez J, Salgado M (2007) Toolpath Selection Based on the Minimum Deflection Cutting Forces in the Programming of Complex Surfaces Milling. International Journal of Machine Tools and Manufacture 47:388-400.

[12] Lauwers B, Lefebvre PP (2006) Five-axis Rough Milling Strategies for Complex Shaped Cavities Based on Morphing Technology. Annals of the CIRP 55(1): 59-62. 